\title{
Single spin asymmetry in forward $p A$ collisions: Phenomenology at RHIC
}

\author{
Sanjin Benic ${ }^{1, *}$ and Yoshitaka Hatta ${ }^{2,1}$ \\ ${ }^{1}$ Yukawa Institute for Theoretical Physics, Kyoto University, Kyoto 606-8502, Japan \\ ${ }^{2}$ Physics Department, Brookhaven National Laboratory, Upton, New York 11973, USA
}

(Received 6 January 2019; published 13 May 2019)

\begin{abstract}
We confront the theoretical result of single spin asymmetry $A_{N}$ in forward $p A$ collisions $p^{\uparrow} A \rightarrow h X$ including the gluon saturation effect with the recent preliminary experimental data from the PHENIX and STAR Collaborations at the Relativistic Heavy Ion Collider. While we find overall reasonable agreement with the STAR data, our results indicate that the strong nuclear suppression of the asymmetry $A_{N} \sim A^{-1 / 3}$ observed by the PHENIX Collaboration cannot be explained within the present understanding of this problem.
\end{abstract}

DOI: 10.1103/PhysRevD.99.094012

\section{INTRODUCTION}

Transverse single spin asymmetries (SSAs), as measured in collisions of an unpolarized probe with a transversely polarized proton, are traditionally a venue to understand the spin structure of the proton [1-3]. For inclusive hadron productions at high $P_{h \perp}, \mathrm{SSA}$ is computed from perturbative QCD where it becomes a probe of collinear twist-3 distributions. Recent measurements at the Relativistic Heavy Ion Collider (RHIC) considered collisions of polarized protons on nuclear targets and so a completely new interplay between spin physics and the physics of gluon saturation becomes a reality [4-11]. This is especially so, as gluon saturation is important in the forward region of the produced hadron where SSA is the largest. Both the STAR [12] and the PHENIX Collaborations [13] reported on preliminary results on SSA in $p^{\uparrow} A \rightarrow h X$ in addition to $p^{\uparrow} p \rightarrow h X$. The PHENIX Collaboration found a striking nuclear suppression $A_{N} \propto A^{-1 / 3}$ with the mass number $A$ in their preliminary datasets. On the other hand, the STAR Collaboration did not find any significant nuclear effect in their data. The two datasets are not necessarily in contradiction to each other, as they are collected for different kinematics. However, the difference in kinematics is actually not very large, and both data are sensitive to the small- $x$ region of the nucleus target. Therefore, it remains a challenge for theorists to explain both data consistently in a single framework.

At first sight, the suppression $A_{N} \propto A^{-1 / 3}$ seems consistent with the prediction of $k_{\perp}$-factorization approaches [4,5]

\footnotetext{
*n leave from Department of Physics, Faculty of Science, University of Zagreb, Bijenička c. 32, 10000 Zagreb, Croatia.

Published by the American Physical Society under the terms of the Creative Commons Attribution 4.0 International license. Further distribution of this work must maintain attribution to the author(s) and the published article's title, journal citation, and DOI. Funded by SCOAP ${ }^{3}$.
}

which include the gluon saturation effects $[14,15]$ in the target nucleus. However, the $k_{\perp}$ factorization does not apply to this process, and a more proper treatment based on the collinear or "hybrid" [9] factorization has identified two contributions with different scaling behaviors $A_{N} \sim$ $\mathcal{O}\left(A^{0}\right)+\mathcal{O}\left(A^{-1 / 3}\right)[16-18]$. The recent fits of the $p^{\uparrow} p$ data $[19,20]$ indicate that the $\mathcal{O}\left(A^{0}\right)$ terms are dominant, so the PHENIX result is actually surprising. Furthermore, the suppression is observed at relatively high values of the hadron $P_{h \perp}$ where one does not expect to see strong nuclear effects at RHIC energies. In view of this, it is premature to link the PHENIX finding with the gluon saturation effect.

In this paper we quantitatively address this problem by numerically computing the SSA in $p^{\uparrow} p \rightarrow h X$ and $p^{\uparrow} A \rightarrow$ $h X$ using the formulas derived in $[16,17]$. We then compare our results with the preliminary STAR and PHENIX data. Following $[19,20]$, we assume that the twist-3 fragmentation contribution is the main cause of SSA in this channel. As for the nucleus, we use the solution of the running coupling Balitsky-Kovchegov (RCBK) equation [21,22]. The main result, presented in Sec. III, shows an overall satisfactory agreement with the preliminary STAR data. On the other hand, we were not able to confirm the nuclear suppression as seen in the PHENIX preliminary results. To the contrary, our results show no nuclear dependence for the PHENIX kinematics, even though we include the saturation effect of the target. We investigate the reason of this failure and discuss what extra contributions are needed to fix this problem.

\section{CROSS SECTION FORMULAS}

Our starting point are the formulas for the spinindependent $p A \rightarrow h X$ cross section and the fragmentation contribution to the spin-dependent $p^{\uparrow} A \rightarrow h X$ cross section within the Color Glass Condensate (CGC) framework. The spin-independent cross section [23] is given as 


$$
\begin{aligned}
\frac{d \sigma}{d^{2} P_{h \perp} d y_{h}}= & \sum_{a} \int_{z_{\min }}^{1} \frac{d z}{z^{2}} x_{q} f_{a}\left(x_{q}, P_{h \perp}^{2}\right) \\
& \times F\left(x_{g}, P_{h \perp} / z\right) D_{h / a}\left(z, P_{h \perp}^{2}\right),
\end{aligned}
$$

where $P_{h \perp}$ and $y_{h}$ are the hadron transverse momenta and rapidity, respectively, with $x_{q, g}=\frac{P_{h \perp}}{z \sqrt{s}} e^{ \pm y_{h}}$ and $z_{\min }=$ $\frac{P_{h \perp}}{\sqrt{s}} e^{y_{h}}$ and we sum over quark flavors as $\sum_{a}$. The function $F\left(x, k_{\perp}\right)$ is the gluon dipole distribution defined as

$$
\begin{aligned}
F\left(x, k_{\perp}\right) & \equiv \pi R_{A}^{2} \int \frac{d^{2} \boldsymbol{x}_{\perp}}{(2 \pi)^{2}} e^{-i \boldsymbol{k}_{\perp} \cdot \boldsymbol{x}_{\perp}} F_{Y}\left(x_{\perp}\right) \\
& \equiv \pi R_{A}^{2} \int \frac{d^{2} \boldsymbol{x}_{\perp}}{(2 \pi)^{2}} e^{-i k_{\perp} \cdot \boldsymbol{x}_{\perp}} \frac{1}{N_{c}}\left\langle\operatorname{Tr}\left[U\left(\boldsymbol{x}_{\perp}\right) U^{\dagger}(0)\right]\right\rangle_{Y},
\end{aligned}
$$

where $Y=\log (1 / x), R_{A}$ is the nuclear radius and $U\left(\boldsymbol{x}_{\perp}\right)$ is the fundamental Wilson line with $\langle\ldots\rangle$ in the third line denoting the color average. In Eq. (1), $f_{a}\left(x, Q^{2}\right)$ is the unpolarized parton distribution function and $D_{h / a}\left(z, Q^{2}\right)$ is the unpolarized hadron fragmentation function evaluated at the scale $Q^{2}=P_{h \perp}^{2}$.

The spin-dependent cross section comes from the quark-gluon-quark contribution, the twist-3 fragmentation contribution and the triple gluon contribution. In this work we consider only the fragmentation contribution as it is the dominant source of SSA in this channel $[19,20]$. We start from the main formula [see Eq. (46) in [17]]

$$
\begin{aligned}
\frac{d \Delta \sigma\left(S_{\perp}\right)}{d^{2} P_{h \perp} d y_{h}}= & \frac{M}{2} S_{\perp i} \epsilon^{i j} \sum_{a} \int_{z_{\min }}^{1} \frac{d z}{z^{2}} x_{q} h_{1}^{a}\left(x_{q}, P_{h \perp}^{2}\right)\left\{-\operatorname{Im} \tilde{e}^{h / a}\left(z, P_{h \perp}^{2}\right) \frac{d}{d\left(P_{h}^{j} / z\right)} F\left(x_{g}, P_{h \perp} / z\right)\right. \\
& \left.+4 \frac{P_{h j}}{P_{h \perp}^{2}} \int_{z}^{\infty} \frac{d z_{1}}{z_{1}^{2}} \frac{z}{\frac{1}{z}-\frac{1}{z_{1}}} \frac{\operatorname{Im} \hat{E}_{F}^{h / a}\left(z_{1}, z, P_{h \perp}^{2}\right)}{N_{c}^{2}-1}\left[\frac{2 \pi N_{c}^{2}}{\pi R_{A}^{2}} \int_{0}^{P_{h \perp} / z_{1}} l_{\perp} d l_{\perp} F\left(x_{g}, l_{\perp}\right)+\frac{1}{z_{1} \frac{1}{z}-\frac{1}{z_{1}}}\right] F\left(x_{g}, P_{h \perp} / z\right)\right\},
\end{aligned}
$$

where $M$ is the proton mass and $S_{\perp}^{i}$ is the proton spin. Here $h_{1}^{a}\left(x, Q^{2}\right)$ is the quark transversity distribution, while $\operatorname{Im} \tilde{e}^{h / a}\left(z, Q^{2}\right)$ and $\operatorname{Im} \hat{E}_{F}^{h / a}\left(z^{\prime}, z, Q^{2}\right)$ are the hadron twist-3 fragmentation functions.

In the next step we approximate $\int_{0}^{P_{h \perp} / z_{1}} d l_{\perp} \simeq \int_{0}^{P_{h \perp} / z} d l_{\perp}$ which is reasonable considering that $z_{1}>z$ while $F\left(x, k_{\perp}\right)$ is a monotonically dropping function of $k_{\perp}$. With this approximation it is possible to make use of the following relations for the twist-3 fragmentation functions:

$$
\begin{aligned}
& \hat{e}_{\overline{1}}^{h / a}\left(z, Q^{2}\right)=z \operatorname{Im} \tilde{e}^{h / a}\left(z, Q^{2}\right)+z \int_{z}^{\infty} \frac{d z^{\prime}}{z^{\prime 2}} P\left(\frac{1}{1 / z^{\prime}-1 / z}\right) \operatorname{Im} \hat{E}_{F}^{h / a}\left(z^{\prime}, z, Q^{2}\right), \\
& \frac{\hat{e}_{\overline{1}}^{h / a}\left(z, Q^{2}\right)}{z}=\frac{1}{2} \frac{d}{d(1 / z)}\left(\frac{\operatorname{Im} \tilde{e}^{h / a}\left(z, Q^{2}\right)}{z}\right)+\frac{1}{z} \int_{z}^{\infty} \frac{d z^{\prime}}{z^{\prime 2}} P\left(\frac{1}{\left(1 / z-1 / z^{\prime}\right)^{2}}\right) \operatorname{Im} \hat{E}_{F}^{h / a}\left(z^{\prime}, z, Q^{2}\right),
\end{aligned}
$$

to eliminate the terms containing the $z^{\prime}$ integral over $\operatorname{Im} \hat{E}_{F}^{h / a}\left(z^{\prime}, z, Q^{2}\right)$ in (3). Here $\hat{e}_{\overline{1}}^{h / a}\left(z, Q^{2}\right)$ is yet another twist-3 fragmentation function. The notation used in this work relates to the notation in Refs. [20,24] as

$$
H^{h / a}\left(z, Q^{2}\right)=-\frac{M_{N}}{M_{h}} \hat{e}_{\overline{1}}^{h / a}\left(z, Q^{2}\right), \quad H^{\perp(1), h / a}\left(z, Q^{2}\right)=\frac{M_{N}}{2 M_{h}} \operatorname{Im} \tilde{e}^{h / a}\left(z, Q^{2}\right), \quad \operatorname{Im} \hat{H}_{F U}^{h / a}\left(z, z^{\prime}, Q^{2}\right)=\frac{M_{N}}{2 M_{h}} \operatorname{Im} \hat{E}_{F}^{h / a}\left(z^{\prime}, z, Q^{2}\right),
$$

with $H^{h / a}\left(z, Q^{2}\right), H^{\perp(1), h / a}\left(z, Q^{2}\right)$, and $\hat{H}_{F U}^{h / a}\left(z, z^{\prime}, Q^{2}\right)$ named as intrinsic, kinematical, and the dynamical twist-3 fragmentation functions, respectively. Equations (4) are known as the QCD equation of motion relation [25,26] and the Lorentz invariance relation [24], respectively. Using (4), Eq. (3) becomes

$$
\begin{aligned}
\frac{d \Delta \sigma\left(S_{\perp}\right)}{d^{2} P_{h \perp} d y_{h}}= & \frac{M}{2} S_{\perp i} \frac{P_{h j}}{P_{h \perp}} \epsilon^{i j} \sum_{a} \int_{z_{\min }}^{1} \frac{d z}{z^{2}} x_{q} h_{1}^{a}\left(x_{q}, P_{h \perp}^{2}\right)\left\{\operatorname{Im} \tilde{e}^{h / a}\left(z, P_{h \perp}^{2}\right) \frac{d}{d\left(P_{h \perp} / z\right)} F\left(x_{g}, P_{h \perp} / z\right)\right. \\
& +\frac{4}{P_{h \perp}} \frac{1}{N_{c}^{2}-1}\left[\frac{2 \pi N_{c}^{2}}{\pi R_{A}^{2}} \int_{0}^{P_{h \perp} / z} l_{\perp} d l_{\perp} F\left(x_{g}, l_{\perp}\right)\left(z \operatorname{Im} \tilde{e}^{h / a}\left(z, P_{h \perp}^{2}\right)-\hat{e}_{\overline{1}}^{a}\left(z, P_{h \perp}^{2}\right)\right)+2 \hat{e}_{\overline{1}}^{h / a}\left(z, P_{h \perp}^{2}\right)\right. \\
& \left.\left.-z \operatorname{Im} \tilde{e}^{h / a}\left(z, P_{h \perp}^{2}\right)-\frac{z}{2} \frac{d}{d(1 / z)}\left(\frac{\operatorname{Im} \tilde{e}^{h / a}\left(z, P_{h \perp}^{2}\right)}{z}\right)\right] F\left(x_{g}, P_{h \perp} / z\right)\right\} .
\end{aligned}
$$


In the following we will numerically compute the SSA defined as

$$
A_{N}=\frac{1}{2} \frac{d \Delta \sigma(\uparrow)-d \Delta \sigma(\downarrow)}{d \sigma},
$$

where in the numerator (denominator) we have the spin-dependent (independent) cross section defined by Eq. (6) [Eq. (1)]. We adopt the convention by which $S_{i} P_{h j} \epsilon^{i j} / P_{h \perp}=\sin \left(\phi_{h}-\phi_{S}\right)=-1$, where $\phi_{h}\left(\phi_{S}\right)$ are azimuthal angles of the outgoing hadron (spin). When the incoming proton is pointing in the $+z$ direction, and with its spin pointing in the $y$ direction, $\Delta \sigma(\uparrow)$ $[\Delta \sigma(\downarrow)=-\Delta \sigma(\uparrow)]$ is the cross section for the hadron emission in the $+x(-x)$ direction or left (right) direction. This explains the "left-right" convention which is also used by STAR and PHENIX.

The nuclear effects are contained in the dipole function $F\left(x, k_{\perp}\right)$ and especially the first term in the spin-dependent cross section (6) depends on the derivative of the dipole. In the saturation regime $\left(k_{\perp} \lesssim Q_{S}\right)$, where $Q_{S}$ is the saturation scale, we would typically get $d F / d k_{\perp} \sim k_{\perp} F / Q_{S}^{2}$. Since the spinindependent cross section (1) goes as $\sim F\left(x, P_{h \perp} / z\right)$, we find, for this particular term, $A_{N} \sim Q_{S}^{-2}$, leading to $A_{N} \sim A^{-1 / 3}$ for the nuclei. Although not immediately obvious, the second term of (6) also scales as $A^{-1 / 3}$ [17]. From a quantitative perspective it is important that the saturation scale in the nuclei scales as $\left(Q_{S}^{A}\right)^{2}=c A^{1 / 3}\left(Q_{S}^{p}\right)^{2}\left(Q_{S}^{p}\right.$ is the saturation scale in the proton) where an additional proportionality factor $c<1$ [27] (in the numerical calculations we will use $c=0.5$ ) will inhibit the overall magnitude of the nuclear suppression. On the other hand, when $k_{\perp} \gg Q_{S}$ we are in the perturbative regime where the dipole distribution has a characteristic dependence $F \sim Q_{S}^{2} / k_{\perp}^{4}$ and so $d F / d P_{h \perp} \sim Q_{S}^{2} / P_{h \perp}^{5}$. The same $Q_{S}$ dependence is found also for the second term in (6): $F / P_{h \perp} \sim Q_{S}^{2} / P_{h \perp}^{5}$ and so in the perturbative limit the nuclear dependence drops out in the ratio.

\section{CALCULATION SETUP AND NUMERICAL RESULTS}

In this section we first explain all the details of our calculation and then we numerically compute SSA and compare with the available preliminary data from STAR and PHENIX. We will often be using the Feynman- $x$ variable: $x_{F}=2 P_{h \perp} \sinh y_{h} / \sqrt{s}$.

For the dipole gluon distributions $F\left(x, k_{\perp}\right)(2)$, we use the numerical solution of the RCBK equation $[21,22]$ from [27]. We take the McLerran-Venugopalan (MV) initial condition at $Y_{0}=\log \left(1 / x_{0}\right)$, where $x_{0}=0.01$ as

$$
F_{Y_{p, A}=Y_{0}}\left(x_{\perp}\right)=\exp \left[-\frac{\left(x_{\perp}^{2}\left(Q_{S, 0}^{p, A}\right)^{2}\right)^{\gamma}}{4} \log \left(\frac{1}{x_{\perp} \Lambda}+e\right)\right] .
$$

Here $Q_{S, 0}^{p, A}$ is the initial saturation scale parameter for the proton and the nuclei, $\gamma$ is the anomalous dimension and $\Lambda$ is the IR cutoff. We use two different parameter sets for the initial condition. Labeling them as set MV and set $\mathrm{MV}^{\gamma}$, the model parameters are MV: $\gamma=1,\left(Q_{S, 0}^{p}\right)^{2}=$ $0.2 \mathrm{GeV}^{2}$ and $\Lambda=0.241 \mathrm{GeV}$ and $\mathrm{MV}^{\gamma}: \gamma=1.119$, $\left(Q_{S, 0}^{p}\right)^{2}=0.168 \mathrm{GeV}^{2}$ and $\Lambda=0.241 \mathrm{GeV}$. For the nuclei, we use the relation $\left(Q_{S, 0}^{A}\right)^{2}=c A^{1 / 3}\left(Q_{S, 0}^{p}\right)^{2}$ with $c=0.5$, as mentioned previously.

For the twist-2 distribution functions $f_{a}\left(x, Q^{2}\right)$, we use the central CTEQ10 set [28]. For the twist-2 pion and kaon fragmentation functions $D_{\pi / a}\left(z, Q^{2}\right)$ and $D_{K / a}\left(z, Q^{2}\right)$ we use the central DSSV set [29]. The transversity distribution $h_{1}^{a}\left(x, Q^{2}\right)$ and the twist-3 pion fragmentation functions $\operatorname{Im} \tilde{e}^{\pi^{+} / a}\left(z, Q^{2}\right)$ are obtained by solving their respective evolution equations numerically with the initial condition determined in [30]. The twist-3 kaon fragmentation function is obtained from [31]. In both cases we employ the Wilczek-Wandzura approximation $\hat{e}_{\overline{1}}^{h / a}\left(z, Q^{2}\right)=$ $z \operatorname{Im} \tilde{e}^{h / a}\left(z, Q^{2}\right)$ as in [30].

In Fig. 1 we show the numerical results of our computation for $A_{N}$ in $p^{\uparrow} p \rightarrow \pi^{0} X$ and $p^{\uparrow} A u \rightarrow \pi^{0} X$ as a function $P_{\pi \perp}$ for several values of $x_{F}$ as compared to the preliminary STAR data [12]. We have used $\left(Q_{S, 0}^{A u}\right)^{2}=3\left(Q_{S, 0}^{p}\right)^{2}$. The full (dashed) lines correspond to the calculation with the MV $\left(\mathrm{MV}^{\gamma}\right)$ model. The shaded band comes from the uncertainty in the transversity and the twist- 3 fragmentation function from the analysis in [30]. In this case, we have calculated $A_{N}$ with the MV model. As a consistency check, we have also computed $A_{N}$ in $p^{\uparrow} p$ using the collinear gluon parton distribution function for the unpolarized proton (as was done in [20]). The result is in good agreement with the one from the RCBK solution shown in Fig. 1. ${ }^{1}$

While the central results, given by the full and dashed lines in Fig. 1, seem to compare well with the overall magnitude of the preliminary STAR data and within its experimental uncertainties, the shaded bands reflect a large theoretical uncertainty in the extraction of the transversity and the twist-3 fragmentation function. Nonetheless, there is a valuable point to be made here regarding the nuclear dependence of $A_{N}$. The nuclear suppression of $A_{N}$ for $p^{\uparrow} A u$ relative to $A_{N}$ in $p^{\uparrow} p$ that is visible in the low- $P_{\pi \perp}$ region is not only the most prominent feature of our calculation, but also quite robust, being of a similar magnitude for the central results as well as for the shaded regions. On the other hand, there are no clear indications of nuclear effects in the STAR data, and in particular there is no nuclear suppression in the low- $P_{\pi \perp}$ region as predicted here by the hybrid factorization framework. The slight hints of nuclear

\footnotetext{
${ }^{1}$ Incidentally, we also confirmed that the forward approximation $\hat{s} \approx-\hat{u} \gg|\hat{t}|$ in the partonic subprocess used to derive formula (3) is actually very good in the kinematics we consider.
} 

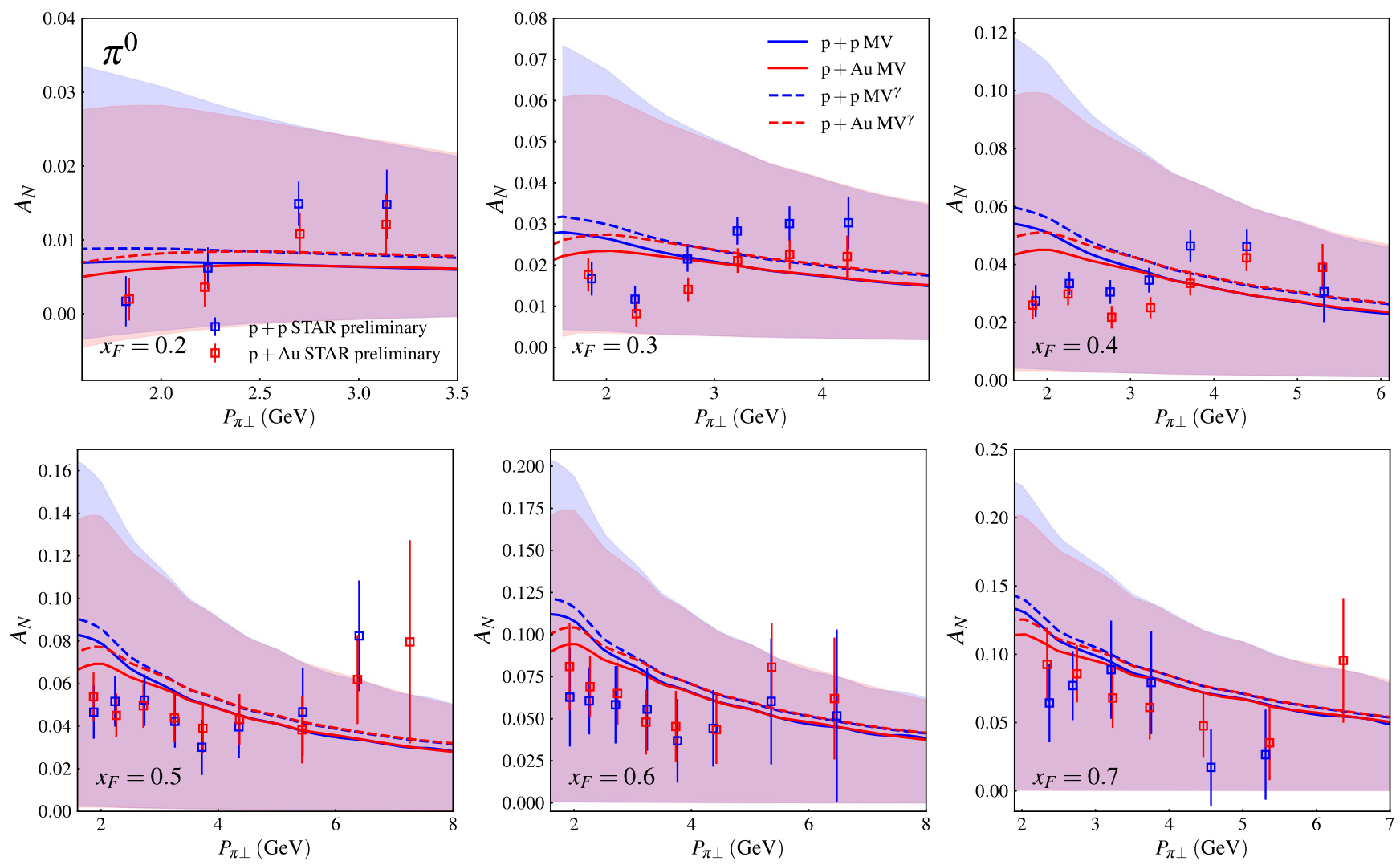

FIG. 1. $\quad A_{N}$ for $p^{\uparrow} p \rightarrow \pi^{0} X$ and $p^{\uparrow} A u \rightarrow \pi^{0} X$ as a function of $P_{\pi \perp}$ at $\sqrt{s}=200 \mathrm{GeV}$ versus preliminary STAR data [12]. The full (dashed) lines are a calculation using the MV $\left(\mathrm{MV}^{\gamma}\right)$ model where we used $\left(Q_{S, 0}^{A u}\right)^{2}=3\left(Q_{S, 0}^{p}\right)^{2}$ as well as the central values for the transversity and the twist-3 fragmentation functions, while the shaded band reflects the uncertainty in extraction of both of these quantities according to [30].

suppression in the higher- $P_{\pi \perp}$ bins, visible in Fig. 1 for $x_{F}=0.3$ and $x_{F}=0.4$ (within errors), and even enhancement for $x_{F}=0.6$ (central values) cannot be reproduced with the present framework either.

In the preliminary PHENIX dataset [13], covering a kinematics range $x_{F} \leq 0.12, A_{N}$ is measured in $p^{\uparrow} p \rightarrow h^{+} X, p^{\uparrow} A l \rightarrow h^{+} X$ and $p^{\uparrow} A u \rightarrow h^{+} X$, where $h^{+}$ is a mixture of outgoing $\pi^{+}$and $K^{+}$. The nuclear dependence of $A_{N}$ in the PHENIX data is most noticeable for the largest measured $x_{F}=0.12$ where also an average $P_{h \perp}=$ $2.9 \mathrm{GeV}$ is measured. At these kinematics, $y_{h}=2.13$ leading to $x_{q}>0.12, x_{g}>0.0017$ and so it is reasonable to apply the forward CGC formulas to compute $A_{N}$. In Fig. 2 we show a comparison of our results for the nuclear dependence of $A_{N}$ in $p^{\uparrow} A \rightarrow h^{+} X^{2}$ to the preliminary PHENIX data, for the kinematics point $x_{F}=0.12$, $P_{h \perp}=2.9 \mathrm{GeV}$. In the PHENIX result $A_{N}$ clearly drops with the increase in the atomic number $A$, and this is consistent with the behavior $A_{N} \sim A^{-1 / 3}$. However, our

\footnotetext{
${ }^{2}$ Even though our numerical calculation includes the contributions of $\pi^{+}$and $K^{+}$, however, quantitatively the $K^{+}$contribution is not more than about $10 \%$ of the full result for $A_{N}$.
}

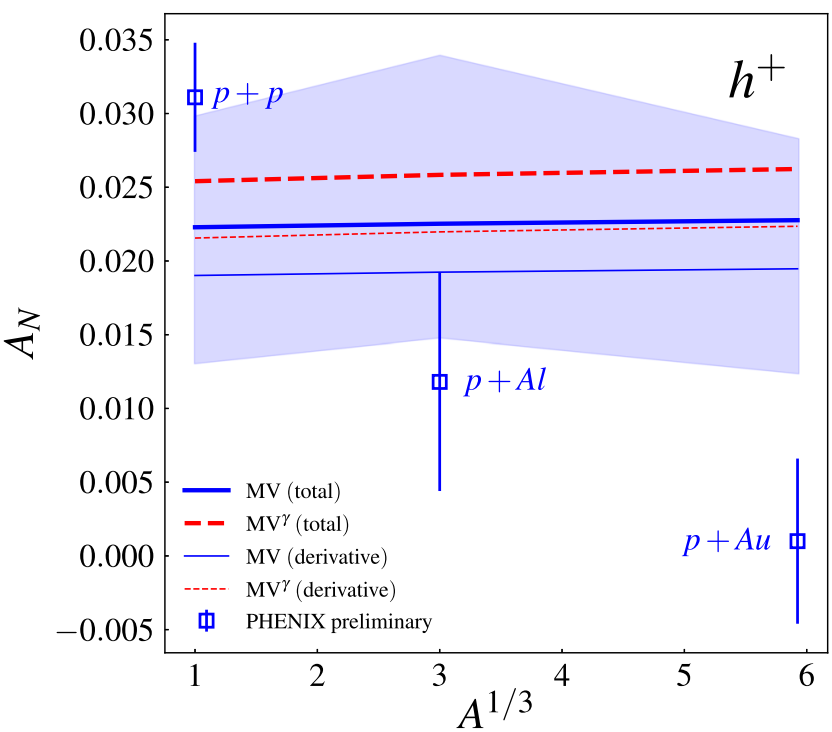

FIG. 2. $A_{N}$ as a function of $A^{1 / 3}$ at $\sqrt{s}=200 \mathrm{GeV}$ versus preliminary data from PHENIX. The thick full (thick dashed) curves represent the MV $\left(\mathrm{MV}^{\gamma}\right)$ model calculation. In the case of the thin curves, we have taken into account only the derivative term in the polarized cross section [first term in Eq. (6)] as a contribution to $A_{N}$. The shaded blue band takes into account the uncertainty of the transversity and the twist-3 fragmentation function according to [30]. 


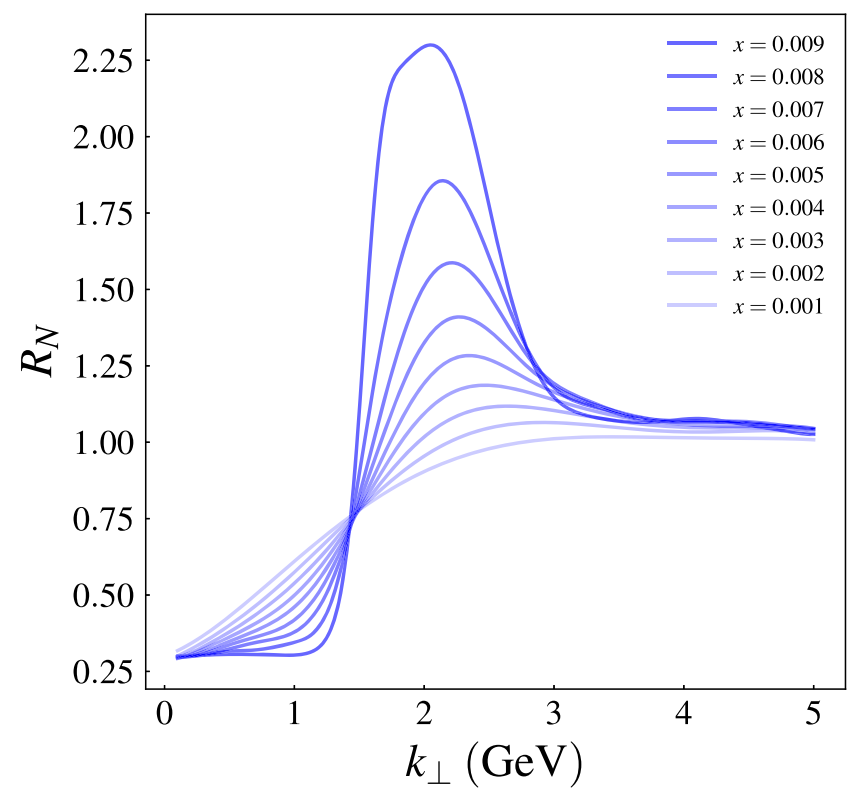

FIG. 3. The quantity $R_{N}$ [see Eq. (9) for the definition] as a function of $k_{\perp}$ for different values of $x$.

current numerical results show virtually no $A$ dependence. The reason is clear: $P_{h \perp}=2.9 \mathrm{GeV}$ is too hard to be sensitive to the saturation scale which is $Q_{S}^{A u} \sim 0.9 \mathrm{GeV}$ for the PHENIX kinematics in the model used here.

To elaborate on this point, let us make an extreme assumption that only the first term of (6) is important. For $P_{h T}<Q_{S}^{A}$, this term is expected to give the scaling $A_{N} \sim$ $A^{-1 / 3}$ and this is demonstrated in Fig. 3 where we plot the double ratio

$$
R_{N} \equiv \frac{\left(d F / d k_{\perp} / F\right)_{A}}{\left(d F / d k_{\perp} / F\right)_{p}}
$$

as a function of $k_{\perp}$ for several values of $x$ using $\left(Q_{S, 0}^{A u}\right)^{2}=$ $3\left(Q_{S, 0}^{p}\right)^{2}$. Close to the initial condition the distribution is nearly Gaussian, and hence the ratio has a plateau in the low- $k_{\perp}$ region at $R_{N} \simeq\left(Q_{S}^{p}\right)^{2} /\left(Q_{S}^{A}\right)^{2} \simeq 1 / 3$. For high $k_{\perp}$, $R_{N} \rightarrow 1$ as a consequence of the perturbative tail. Going lower in $x$ via the RCBK equation, the peak position moves toward the high- $k_{\perp}$ region and the plateau shrinks - already for $x \sim 0.001$ the value of $1 / 3$ is reached only for very small $k_{\perp}$. For $k_{\perp}$ as large as $2.9 \mathrm{GeV}, R_{N}$ never deviates significantly from unity. Therefore, even in this extreme scenario $A_{N}$ does not have nuclear dependence. This is also illustrated by the thin curves in Fig. 2 where we computed $A_{N}$ including only the derivative term in the numerator. Note that we have only included the fragmentation contribution, but it is clear that adding the contribution from the twist-3 quark-gluon distributions [16] will not help resolve this issue.

\section{CONCLUSIONS}

We have made a numerical computation of SSA in $p^{\uparrow} p$ and $p^{\uparrow} A$ in the forward region including the gluon saturation effect of the nucleus. Using the current stateof-the-art twist-3 fragmentation functions and the dipole gluon distribution, we compared our results to the preliminary STAR and PHENIX data. While the saturation-based description seems to describe well the overall magnitude of the STAR data, it fails to explain the scaling $A_{N} \sim A^{-1 / 3}$ observed by the PHENIX Collaboration.

According to the result of $[16,17]$, a strong nuclear suppression of $A_{N}$ is possible only if the $\sim A^{-1 / 3}$ terms dominate over the $\sim A^{0}$ terms, and if one looks at $P_{h \perp}$ less than $Q_{S}$. The recent fit of the $p p$ data [20] suggests that the first condition does not hold, and the second condition is also violated by the high value of $P_{h \perp}$ measured by the PHENIX Collaboration. This makes the PHENIX result all the more striking. It is also puzzling that there seems to be a sudden change in the behaviors of $A_{N}$ between $x_{F}=0.2$ (the lowest value measured by the STAR Collaboration) and $x_{F}=0.12$ (highest value measured by the PHENIX Collaboration).

This may call for alternative mechanisms of SSA around $x_{F} \sim 0.1$ whose nuclear dependence comes from a different source. Indeed the region $0.1 \lesssim x_{F} \lesssim 0.2$ might be special—it is roughly the "threshold" region where $A_{N}$ starts to grow. Thus the value of $A_{N}$ itself is very small in this region and a small effect can cause a large numerical impact. Perhaps one should include not only the $q+g$ channel (as we did in this paper), but also the $g+g$ channel. Since there is no "gluon transversity" distribution, the fragmentation contribution is absent in this channel, but the collinear three-gluon or "odderon" contribution comes into play [7,10,32-34]. A precise evaluation of SSA including these effects appears to be a challenging task.

\section{ACKNOWLEDGMENTS}

We thank Carl Gagliardi, and Jeongsu Bok and Jin Huang for sharing with us the preliminary STAR and PHENIX data, respectively. We thank Kevin Dusling for tables of the RCBK solution and Alexey Prokudin for transversity and fragmentation functions as well as the clarifications of the uncertainty analysis. We also thank Elke Aschenauer for comment on uncertainty in transversity and fragmentation functions. Y. H. is supported by the U.S. Department of Energy, Office of Science, Office of Nuclear Physics, under Contracts No. de-sc0012704. S. B. is supported by a Japanese Society for the Promotion of Science (JSPS) postdoctoral fellowship for foreign researchers under Grant No. 17F17323. S. B. also acknowledges Hrvatska Zaklada za Znanost (HRZZ) Grant No. 8799 for computational resources. 
[1] U. D’Alesio and F. Murgia, Prog. Part. Nucl. Phys. 61, 394 (2008).

[2] D. Boer et al., arXiv:1108.1713.

[3] M. Grosse Perdekamp and F. Yuan, Annu. Rev. Nucl. Part. Sci. 65, 429 (2015).

[4] D. Boer, A. Dumitru, and A. Hayashigaki, Phys. Rev. D 74, 074018 (2006).

[5] Z.-B. Kang and F. Yuan, Phys. Rev. D 84, 034019 (2011).

[6] Z.-B. Kang and B.-W. Xiao, Phys. Rev. D 87, 034038 (2013).

[7] Y. V. Kovchegov and M. D. Sievert, Phys. Rev. D 86, 034028 (2012); 86, 079906(E) (2012).

[8] T. Altinoluk, N. Armesto, G. Beuf, M. Martínez, and C. A. Salgado, J. High Energy Phys. 07 (2014) 068.

[9] A. Schäfer and J. Zhou, Phys. Rev. D 90, 034016 (2014).

[10] J. Zhou, Phys. Rev. D 92, 014034 (2015).

[11] D. Boer, M. G. Echevarria, P. Mulders, and J. Zhou, Phys. Rev. Lett. 116, 122001 (2016).

[12] C. Dilks (STAR Collaboration), Proc. Sci., DIS2016 (2016) 212.

[13] C. Aidala et al. (PHENIX Collaboration), arXiv:1903.07422.

[14] E. Iancu and R. Venugopalan, The Color glass condensate and high-energy scattering in QCD, in Quark-Gluon Plasma 4, edited by R. C. Hwa and X.-N. Wang (World Scientific Publishing, Singapore, 2004), p. 249.

[15] F. Gelis, E. Iancu, J. Jalilian-Marian, and R. Venugopalan, Annu. Rev. Nucl. Part. Sci. 60, 463 (2010).

[16] Y. Hatta, B.-W. Xiao, S. Yoshida, and F. Yuan, Phys. Rev. D 94, 054013 (2016).

[17] Y. Hatta, B.-W. Xiao, S. Yoshida, and F. Yuan, Phys. Rev. D 95, 014008 (2017).
[18] J. Zhou, Phys. Rev. D 96, 034027 (2017).

[19] K. Kanazawa, Y. Koike, A. Metz, and D. Pitonyak, Phys. Rev. D 89, 111501 (2014).

[20] L. Gamberg, Z.-B. Kang, D. Pitonyak, and A. Prokudin, Phys. Lett. B 770, 242 (2017).

[21] I. Balitsky, Nucl. Phys. B463, 99 (1996).

[22] Y. V. Kovchegov, Phys. Rev. D 60, 034008 (1999).

[23] A. Dumitru, A. Hayashigaki, and J. Jalilian-Marian, Nucl. Phys. A765, 464 (2006).

[24] K. Kanazawa, Y. Koike, A. Metz, D. Pitonyak, and M. Schlegel, Phys. Rev. D 93, 054024 (2016).

[25] Z.-B. Kang, F. Yuan, and J. Zhou, Phys. Lett. B 691, 243 (2010).

[26] A. Metz and D. Pitonyak, Phys. Lett. B 723, 365 (2013); 762, 549(E) (2016).

[27] K. Dusling, F. Gelis, T. Lappi, and R. Venugopalan, Nucl. Phys. A836, 159 (2010).

[28] H.-L. Lai, M. Guzzi, J. Huston, Z. Li, P. M. Nadolsky, J. Pumplin, and C.-P. Yuan, Phys. Rev. D 82, 074024 (2010).

[29] D. de Florian, R. Sassot, M. Epele, R. J. Hernández-Pinto, and M. Stratmann, Phys. Rev. D 91, 014035 (2015).

[30] Z.-B. Kang, A. Prokudin, P. Sun, and F. Yuan, Phys. Rev. D 93, 014009 (2016).

[31] M. Anselmino, M. Boglione, U. D’Alesio, J. O. Gonzalez Hernandez, S. Melis, F. Murgia, and A. Prokudin, Phys. Rev. D 93, 034025 (2016).

[32] Y. Koike and S. Yoshida, Phys. Rev. D 84, 014026 (2011).

[33] Y. Koike, K. Tanaka, and S. Yoshida, Phys. Rev. D 83, 114014 (2011).

[34] Y. Hatta, K. Kanazawa, and S. Yoshida, Phys. Rev. D 88, 014037 (2013). 